\title{
Estimativa da incerteza de medição na determinação de elementos maiores e menores em rochas silicáticas por espectrometria de fluorescência de raios $X$ com resultados de controle de qualidade
}

\section{Jacinta Enzweiler* \\ Maria Aparecida Vendemiatto}

Instituto de Geociências, Universidade Estadual de Campinas (UNICAMP) - Campinas (SP), Brasil.

*Autor correspondente: jacinta@ige.unicamp.br

\section{Resumo}

A incerteza da medição expressa a exatidão de um resultado analítico. Uma forma prática de estimar a incerteza de medição é utilizar séries de resultados obtidos ou pela análise de materiais de referência certificados (MRC) ou da participação em testes de proficiência. As duas abordagens foram utilizadas para estimar as incertezas associadas à determinação de frações de massa de óxidos de elementos maiores e menores em silicatos por espectrometria de fluorescência de raios X (FRX). Trinta porções-teste do MRC BRP-1 (Basalto Ribeirão Preto) foram preparadas como discos de vidro e analisadas de acordo com um procedimento de rotina. Com os resultados, foi calculada a incerteza-padrão relativa, resultante dos erros aleatórios. Esta foi combinada à incerteza de tendência que expressa o erro sistemático e foi calculada com os dados de tendência relativa, a incerteza-padrão relativa da média dos resultados e a incerteza do valor de referência do MRC. A incerteza combinada foi expandida com fator de abrangência $\mathrm{k}=2$ para obter a incerteza de medição (U\%).

$\mathrm{Na}$ segunda abordagem, a incerteza de tendência foi obtida com resultados de dez rodadas do Teste de Proficiência GeoPT ${ }^{\mathrm{TM}}$. Esta abrange um intervalo composicional mais amplo e resultou em maiores incertezas de medição que as obtidas com os resultados do MRC. As incertezas de medição calculadas foram comparadas a uma hipotética incerteza-alvo, dada por um terço da reprodutibilidade entre laboratórios e calculada pela expressão de Horwitz. Os dados indicam que incertezas robustas associadas às medições de rotina seriam mais adequadamente estimadas analisando vários MRC para cobrir as frações de massa de interesse da matriz.

Palavras-chave: silicatos, geoquímica analítica, incerteza, fluorescência de raios $\mathrm{X}$, materiais de referência.

\section{Abstract}

The measurement uncertainty expresses the accuracy of an analytical result. A practical way of estimating the measurement uncertainty is using series of results obtained either from the analysis of certified reference materials (CRM) or the participation in proficiency tests. Both approaches were used to estimate the uncertainties associated to the determination of mass fractions of oxides of major and minor elements in silicates by X-ray fluorescence spectrometry (XRF). Thirty 
test portions of CRM BRP-1 (Basalt Ribeirão Preto) were prepared as glass disks and analyzed in a routine procedure. The results were used to calculate the relative standard uncertainty, resultant from random errors. This was combined with the uncertainty of bias that accounts for the systematic error and is calculated with data of relative bias, relative standard uncertainty of the mean of the analytical results and the uncertainty of the reference value of the CRM. The combined uncertainty was expanded to coverage factor $k=2$ to obtain the measurement uncertainty $(U \%)$.

In the second approach, the uncertainty of bias was obtained from results of ten rounds of the Proficiency Test GeoPT TM. This covers a wider range of compositions and resulted in larger measurement uncertainties compared to those obtained with the CRM results. The calculated measurement uncertainties were compared with a hypothetic target measurement uncertainty, given by one third of the reproducibility between laboratories as calculated by the Horwitz expression. The data indicate that robust uncertainties associated to routine measurements would be better estimated by analyzing several CRM to cover the mass fractions of interest of the matrix under consideration.

Keywords: silicates, analytical geochemistry, uncertainty, X-ray fluorescence, reference materials.

\section{INTRODUÇÃO}

Resultados analíticos acompanhados das respectivas incertezas de medição dão indicação da sua qualidade, ajudam na interpretação dos dados e na tomada de decisões (EURACHEM/CITAC Guide CG 4 2012). A incerteza de medição é um parâmetro associado a cada mensurando e depende do método de medição, da matriz e do laboratório e deve atender ao uso pretendido dos resultados de medição. Laboratórios acreditados ou que almejam acreditação necessariamente devem utilizar métodos validados e que tenham a incerteza de medição declarada. Laboratórios de pesquisa são mais dinâmicos e raramente são acreditados, mas mesmo assim devem ter rotinas de controle e garantia de qualidade (Adams et al. 1998).

Alguns conceitos relevantes de metrologia, definidos no Vocabulário Internacional de Metrologia (VIM3, 2012), são apresentados na Tabela 1 conforme sua relação mútua didaticamente proposta por Menditto et al. (2007). O detalhamento dos conceitos da Tabela 1 será omitido, mas as notas explicativas de cada um deles (VIM3 2012) são relevantes. Por exemplo, a exatidão, a veracidade e a precisão não são grandezas e, portanto, não são expressas numericamente. A veracidade e a precisão juntas resultam na exatidão de medição e são expressas, respectivamente, pela tendência, desvio-padrão (ou coeficiente de variação) e incerteza de medição. A incerteza de medição de ensaios analíticos resulta da combinação dos erros aleatórios e sistemáticos. A estimativa de incerteza de medição permite identificar as maiores fontes de incerteza e avaliar se elas devem e podem ser diminuídas para conferir maior robustez aos resultados ou atender ao uso pretendido dos mesmos. Os fundamentos sobre a incerteza de medição e a sua quantificação a partir de duas categorias, isto é, de incertezas do Tipo A e do Tipo B, são encontrados em guias internacionais atualizados regularmente (ISO Guide 98-3, 2008, EURACHEM/CITAC Guide CG 4 2012). Este guia traz a abordagem também conhecida como bottom-up, que consiste em avaliar as fontes de incerteza do método analítico passo a passo, isto é, identificá-las, quantificá-las e combiná-las (Kirkup \& Frenkel 2006, Hibbert 2007). Este procedimento é trabalhoso e nem sempre todas as fontes de incerteza são corretamente incluídas na avaliação.

Algumas abordagens alternativas, conhecidas como top down, podem ser úteis para quantificar a incerteza de medição. Elas baseiam-se na utilização de informações disponíveis de controle de qualidade e/ou de validação de métodos analíticos (e.g., Magnusson et al. 2008), tais como dados de precisão e de veracidade. Os dados de precisão podem ser o desvio-padrão obtido pela análise de duplicatas de certo número de amostras de rotina ou ainda de replicatas de uma ou mais amostras, analisadas em condições de precisão intermediária ou reprodutibilidade interna do laboratório. Os dados de tendência de medição são os obtidos pela análise de materiais de referência com valores atribuídos. Se disponíveis, os dados de tendência podem ser os resultados de estudos interlaboratoriais ou de testes de proficiência. Neste caso, Magnusson et al. (2008) recomendam que o laboratório deve ter resultados de pelo menos seis rodadas de teste de proficiência, realizadas dentro do intervalo de tempo razoável para estimar a incerteza de tendência.

O objetivo deste trabalho foi estimar a incerteza de medição de elementos maiores e menores em rochas silicáticas, determinados por fluorescência de raios X (FRX), usando as abordagens top-down de quantificação das incertezas de medição acima mencionadas, isto é, com os resultados analíticos de controle de qualidade previamente obtidos no laboratório. As incertezas obtidas pelos dois procedimentos são comparadas entre si, bem como com os valores de reprodutibilidade entre laboratórios, conforme calculados por uma das fórmulas de Horwitz que relacionam o desvio-padrão relativo com a concentração (Horwitz et al. 1980; Hibbert 2007). 


\begin{tabular}{|c|c|c|c|c|}
\hline Tipo de erro & & $\begin{array}{c}\text { Características de desem- } \\
\text { penho }\end{array}$ & & $\begin{array}{c}\text { Expressão quantitativa } \\
\text { das características de } \\
\text { desempenho }\end{array}$ \\
\hline $\begin{array}{c}\text { Erro sistemático } \\
\text { Componente do erro de } \\
\text { medição que, em medi- } \\
\text { ções repetidas, permane- } \\
\text { ce constante ou varia de } \\
\text { maneira previsível. }\end{array}$ & $\rightarrow$ & $\begin{array}{l}\text { Veracidade de medição } \\
\text { Grau de concordância } \\
\text { entre a média dum nú- } \\
\text { mero infinito de valores } \\
\text { medidos repetidos e um } \\
\text { valor de referência. }\end{array}$ & $\rightarrow$ & $\begin{array}{c}\text { Tendência de medição } \\
\text { Estimativa dum erro siste- } \\
\text { mático. }\end{array}$ \\
\hline$\downarrow$ & & $\downarrow$ & & $\downarrow$ \\
\hline $\begin{array}{l}\text { Erro total } \\
\text { Diferença entre o valor } \\
\text { medido duma grandeza } \\
\text { e um valor de referência. }\end{array}$ & $\rightarrow$ & $\begin{array}{l}\text { Exatidão de medição } \\
\text { Grau de concordância } \\
\text { entre um valor medido e } \\
\text { um valor verdadeiro dum } \\
\text { mensurando. }\end{array}$ & $\rightarrow$ & $\begin{array}{c}\text { Incerteza de medição } \\
\text { Parâmetro não negativo } \\
\text { que caracteriza a disper- } \\
\text { são dos valores atribuídos } \\
\text { a um } \\
\text { mensurando, com base } \\
\text { nas informações utiliza- } \\
\text { das. }\end{array}$ \\
\hline$\uparrow$ & & $\uparrow$ & & $\uparrow$ \\
\hline $\begin{array}{l}\text { Erro aleatório } \\
\text { Componente do erro de } \\
\text { medição que, em medi- } \\
\text { ções repetidas, varia de } \\
\text { maneira imprevisível. }\end{array}$ & $\rightarrow$ & $\begin{array}{l}\text { Precisão de medição } \\
\text { Grau de concordância } \\
\text { entre indicações ou valo- } \\
\text { res medidos, obtidos por } \\
\text { medições repetidas, } \\
\text { no mesmo objeto ou em } \\
\text { objetos similares, sob con- } \\
\text { dições especificadas. }\end{array}$ & $\rightarrow$ & $\begin{array}{l}\text { Desvio-padrão } \\
\text { Repetibilidade/ } \\
\text { precisão intermediária/ } \\
\text { reprodutibilidade }\end{array}$ \\
\hline
\end{tabular}

\section{Tabela 1}

Relação entre os tipos gerais de erros, as respectivas características de desempenho e os termos usados para expressá-las quantitativamente e definições conforme VIM3 (2012).

(Adaptado de Menditto et al. 2007).

\section{MATERIAIS E MÉTODOS}

Dois métodos de estimativa de incerteza de medição foram utilizados. No primeiro, somente os resultados obtidos para o material de referência certificado (MRC) BRP-1, (Basalto Ribeirão Preto, Cotta et al. 2008 ) analisado como uma amostra de rotina foram empregados. No segundo, foram utilizados os dados da participação no Programa Teste de Proficiência para Laboratórios de Geoquímica Analítica $\mathrm{GeoPT}^{\mathrm{TM}}$, organizado pela International Association of Geoanalysts (IAG) e realizado desde 1996, com a distribuição anual de duas amostras cominuídas aos laboratórios participantes. O Laboratório de Geoquímica do IG/UNICAMP participa do programa desde a primeira rodada, o GeoPT1 (Thompson et al. 1996).

Os resultados analíticos foram obtidos pelo método de rotina utilizado para determinar elementos maiores e menores em rochas silicáticas por espectrometria de fluorescência de raios X. Na análise, foram empregados discos de vidro das amostras pulverizadas. A preparação física das amostras e a etapa seguinte da preparação dos discos de vidro utilizados nas determinações quase sempre são realizadas pelo próprio usuário, orientado por um técnico do laboratório. A cada lote de 20 amostras, o usuário prepara pelo menos uma duplicata das próprias amostras desde a etapa do seu quarteamento. $\mathrm{Na}$ fase de preparação dos discos de vidro, é incluído também um material de referência para controle de qualidade.
Os discos de vidro usados nas medições foram preparados com uma porção-teste da amostra $(1,0000 \mathrm{~g})$ misturada e fundida com 6,0000 g de metaborato e tetraborato de lítio $(50 / 50 \mathrm{~m} / \mathrm{m})$ (Claisse, Canadá), após a secagem de ambos em estufa a $105^{\circ} \mathrm{C}$ por pelo menos duas horas e ambientação térmica em dessecador. A amostra e o fundente foram pesados diretamente em cadinho de liga $\mathrm{Pt} / \mathrm{Au}$ (95/5) e homogeneizados com uma pequena espátula de plástico. Duas gotas de solução aquosa de iodeto de lítio $(250 \mathrm{~g} / \mathrm{L})$ foram adicionadas e a fusão foi realizada num equipamento de fusão (Fluxy 300, Claisse, Canadá). Após o término do processo, o fundido foi automaticamente vertido em molde de $\mathrm{Pt} / \mathrm{Au}$ e, no final do resfriamento controlado, foi produzido um disco de vidro com $30 \mathrm{~mm}$ de diâmetro e $3 \mathrm{~mm}$ espessura.

As análises foram realizadas no método de rotina utilizado para determinar elementos maiores e menores em rochas silicáticas num espectrômetro PW2404 (PANalytical, ex-Philips, Holanda). O método é semelhante ao anteriormente descrito (Vendemiatto \& Enzweiler 2001), com pequenas modificações nos parâmetros instrumentais e com 39 calibradores. Dentre estes, há principalmente materiais de referência de rochas silicáticas, mas também alguns óxidos puros $\left(\mathrm{SiO}_{2}, \mathrm{Al}_{2} \mathrm{O}_{3}\right.$ e $\left.\mathrm{Fe}_{2} \mathrm{O}_{3}\right)$ ou a mistura destes, todos preparados como vidros conforme o procedimento acima. A calibração do método analítico incluindo a correção de efeitos de matriz baseia-se nos procedimentos disponíveis no software Super Q (PANalytical B.V.). 


\subsection{Cálculo da incerteza de medição com resultados analíticos de um material de referência certificado}

Trinta resultados obtidos ao longo de dois anos para o MRC BRP-1 durante as análises de rotina foram utilizados. Os dados foram tratados conforme proposto por Magnusson et al. (2008) e a Tabela 2 traz todas as etapas dos cálculos da incerteza para dióxido de silício $\left(\mathrm{SiO}_{2}\right)$.

$\mathrm{O}$ valor certificado da fração em massa de $\mathrm{SiO}_{2}$ no MRC BRP-1 é $50,39 \pm 0,15 \%(\mathrm{~m} / \mathrm{m})$, onde a incerteza corresponde ao fator de abrangência $\mathrm{k}=2$, isto é, com intervalo de confiança próximo a $95 \%$. O valor médio dos resultados analíticos para $\mathrm{SiO}_{2}$ foi $50,43 \pm 0,28 \%$ onde a incerteza corresponde ao desvio-padrão dos 30 dados, com coeficiente de variação de $0,56 \%$. Todas as incertezas calculadas na Tabela 2 são relativas, isto é, dadas em percentagem.
A etapa 1 consiste em especificar o mensurando e uma meta de incerteza expandida. A incerteza-alvo proposta de $1,5 \%$ foi calculada como $1 / 3$ da reprodutibilidade esperada entre laboratórios, conforme calculada pela expressão de Horwitz $\sigma_{\mathrm{H}}=0,02 \mathrm{x}^{0,8495}$, expandida para $\mathrm{k}=2$, onde $\sigma_{\mathrm{H}} \mathrm{e}$ $\mathrm{x}$ correspondem aos valores absolutos desvio-padrão e de fração em massa do componente no material. A fração $1 / 3$ desta expressão foi escolhida por ser menor que o valor $1 / 2 \sigma_{\mathrm{H}}$ usado pelos organizadores do programa GeoP $T^{\mathrm{TM}}$, para avaliar os resultados de laboratórios que fornecem dados para uso em litogeoquímica e, também, por ser o critério de incerteza máxima proposto para os valores de referência de MR certificados segundo o protocolo da IAG (Kane et al. 2003).

\begin{tabular}{|c|c|c|}
\hline Etapa & Ação & Exemplo: $\mathrm{SiO}_{2}$ em rochas silicáticas por FRX \\
\hline 1 & $\begin{array}{l}\text { Especificar mensu- } \\
\text { rando }\end{array}$ & $\begin{array}{c}\text { Determinação de } \mathrm{SiO}_{2} \text { em rochas silicáticas por FRX em discos de } \\
\text { vidro. Incerteza-alvo }<1,5 \% .\end{array}$ \\
\hline 2 & $\begin{array}{l}\text { Quantificar a } \\
\text { incerteza de repro- } \\
\text { dutibilidade } u(R w) \\
\text { com a } \\
\text { amostra controle }\end{array}$ & $\begin{array}{l}\text { A amostra controle MRC BRP-1 forneceu } \mathrm{SiO}_{2}=50,43 \pm 0,28 \%(\mathrm{~m} / \mathrm{m}) \\
\text { onde a incerteza é o desvio-padrão de } \mathrm{n}=30 \text {. A análise inclui todas as } \\
\text { etapas após a pulverização da amostra. } \\
\qquad u(R w)=100 * 0,28 / 50,43=0,56 \%\end{array}$ \\
\hline 3 & $\begin{array}{l}\text { Quantificar a ten- } \\
\text { dência do método } \\
\text { e do laboratório }\end{array}$ & $\begin{array}{l}\text { O MRC possui valor certificado de } \mathrm{SiO}_{2}=50,39 \pm 0,15 \% \\
\quad \text { (incerteza: } U(\text { Cref })) \\
\text { Tendência relativa: tend }=100 \times(50,43-50,39) / 50,39=0,086 \% \\
\mathrm{u}(\text { Cref })_{\text {absoluta }}=0,15 / 2=0,075 \% \\
\mathrm{u}(\text { Cref })_{\text {relativa }}=100 \times 0,075 / 50,39=0,15 \%\end{array}$ \\
\hline \multirow[t]{2}{*}{4} & $\begin{array}{l}\text { Converter para } \\
\text { incerteza-padrão } \\
u(x) \text { e obter a incer- }\end{array}$ & $u(x)=u(R w)=0,56 \% ; s_{\text {tend }}=0,56 \%(\mathrm{n}=30)$ \\
\hline & $\begin{array}{l}u(x) \text { e obter a Incer- } \\
\text { teza de tendência } \\
u_{\text {tend }}\end{array}$ & $u_{\text {tend }}=\sqrt{(\text { tend })^{2}+\left(\frac{s_{\text {tend }}}{\sqrt{n}}\right)^{2}+[u(\text { Cref })]^{2}}=\sqrt{0,086^{2}+\left(\frac{0,56}{\sqrt{30}}\right)^{2}+0,15^{2}}=0,20 \%$ \\
\hline 5 & $\begin{array}{l}\text { Calcular a incerte- } \\
\text { za combinada }\end{array}$ & $u_{c}=\sqrt{u(x)^{2}+u_{\text {tend }}^{2}}=\sqrt{0,56^{2}+0,20^{2}}=0,60 \%$ \\
\hline 6 & $\begin{array}{l}\text { Calcular a incerte- } \\
\text { za expandida } \\
U=2 \cdot u_{c}\end{array}$ & $U_{M R C}=2 \times 0,60=1,2 \%$ \\
\hline
\end{tabular}

MRC: material de referência certificado; FRX: fluorescência de raios $X$

\subsection{Cálculo da incerteza de medição com resultados analíticos obtidos na participação do Teste de Proficiência GeoPT ${ }^{\mathrm{TM}}$}

No procedimento descrito acima, um MRC foi utilizado para obter a componente da incerteza da tendência. Para avaliar a influência da variabilidade composicional das amostras de rotina analisadas, foram utilizados os resultados obtidos pelo laboratório na participação em dez rodadas do Teste de Proficiência GeoPT $T^{\mathrm{TM}}$ como segundo método para quantificar a incerteza de tendência.

Os laboratórios participantes do programa internacional de teste de proficiência GeoP $T^{\mathrm{TM}}$ recebem amostras pulverizadas e homogêneas de matriz geológica, para análise segundo métodos e condições de rotina. Os resultados são avaliados estatisticamente pelos organizadores e um escore Z é calculado para cada dado submetido pelos laboratórios participantes. O relatório final de desempenho dos participantes informa o escore $\mathrm{Z}$ para cada dado fornecido e, se este estiver no intervalo de \pm 2 , o resultado é considerado satisfatório. Em cada rodada do teste de proficiência são apresentados resultados estatísticos como o valor atribuído $\left(\mathrm{X}_{\mathrm{a}}\right)$, incerteza do valor atribuído (sdm), o valor alvo de Horwitz $\left(\mathrm{H}_{\mathrm{a}}=\sigma_{\mathrm{H}}\right)$, entre outras informações.

O cálculo da incerteza de tendência foi realizado com os resultados das rodadas de número 19 a 29 do teste de proficiência GeoPT, que contemplam as seguintes amostras, respectivamente: gabro MGR-N (GeoPT19); rocha ultramáfica, OPY-1 (GeoPT20); granito, MGT-1 (GeoPT21); basalto MBL-1 (GeoPT22); pegmatito OU-9 (GeoPT23); grauvaca OU-10 (GeoPT24); basalto HTB-1 (GeoPT 25); andesito MGL-AND (GeoPT27); folhelho SBC-1 (GeoPT28) e nefilinito NKT-1 (GeoPT29). Os resultados do GeoPT26, cuja amostra foi do cimento Portland OPC-1, não foram utilizados 
na quantificação de incerteza. Em poucos casos isolados, na ausência de valor atribuído para algum constituinte, ou ainda do escore fora do intervalo \pm 2 , foram utilizados resultados de GeoPT anteriores. Os dados utilizados compreendem os seguintes intervalos de frações de massa dos óxidos dos elementos maiores e menores $[\%(\mathrm{~m} / \mathrm{m})]: \mathrm{SiO}_{2}(37,78-79,50) ; \mathrm{TiO}_{2}$ $(0,057-3,85) ; \mathrm{Al}_{2} \mathrm{O}_{3}(7,95-21,00) ; \mathrm{Fe}_{2} \mathrm{O}_{3}(0,74-15,54) ; \mathrm{MnO}$ $(0,060-0,215) ; \mathrm{MgO}(0,380-21,43) ; \mathrm{CaO}(0,29-15,29)$; $\mathrm{Na}_{2} \mathrm{O}(0,68-4,48) ; \mathrm{K}_{2} \mathrm{O}(0,19-4,67)$ e $\mathrm{P}_{2} \mathrm{O}_{5}(0,03-0,92)$. Aqui e em todo o texto, $\mathrm{Fe}_{2} \mathrm{O}_{3}$ refere-se ao ferro total assim expresso.

A Tabela 3 apresenta as etapas do cálculo da incerteza de tendência para $\mathrm{SiO}_{2}$ com os resultados de medições das amostras do $\mathrm{GeoPT}$ e os respectivos valores atribuídos. Os dados de incerteza da reprodutibilidade do método e do laboratório $u(R w)$ obtidos para o MRC BRP-1 foram usados novamente (etapa 2). A incerteza da tendência foi calculada conforme indicado nas etapas 3 e 4 da Tabela 3. As incertezas foram combinadas (etapa 5) e expandidas (etapa 6) para obter a incerteza de medição relativa $\mathrm{U}_{\mathrm{TP}}$

\begin{tabular}{|c|c|c|}
\hline Etapa & Ação & Exemplo: $\mathrm{SiO}_{2}$ em rochas silicáticas por FRX \\
\hline 1 & $\begin{array}{l}\text { Especificar men- } \\
\text { surando }\end{array}$ & $\begin{array}{c}\text { Determinação de } \mathrm{SiO}_{2} \text { em rochas silicáticas por FRX em } \\
\text { discos de vidro. Incerteza-alvo }<1,5 \% .\end{array}$ \\
\hline 2 & $\begin{array}{l}\text { Quantificar } u(R w) \\
\text { com } \\
\text { amostra controle }\end{array}$ & $\begin{array}{c}\text { A amostra controle é o MRC BRP-1 que fornece } s=0,28 \\
\text { para } \mathrm{SiO}_{2}=50,43 \%(m / m) \text { e } u(x)=u(R w)=0,56 \% \\
\text { A análise inclui todas as etapas após a pulverização da } \\
\text { amostra. }\end{array}$ \\
\hline 3 & $\begin{array}{l}\text { Quantificar a ten- } \\
\text { dência do método } \\
\text { e do laboratório }\end{array}$ & $\begin{array}{l}\text { Participação em } 10 \text { rodadas de teste de proficiência com } \\
\text { matriz semelhante, mas com intervalo amplo de fração } \\
\text { em massa. Tendência relativa média para } \\
\qquad \mathrm{n}=10: \mathrm{RMS}_{\text {tend }}=\sqrt{\frac{\sum(\text { tend })^{2}}{n}}=0,51 \%\end{array}$ \\
\hline 4 & $\begin{array}{c}\text { Converter os } \\
\text { componentes à } \\
\text { incerteza-padrão } \\
u(x)\end{array}$ & $\begin{array}{c}u\left(C_{r e f}\right)=s d m=\frac{H a}{\sqrt{n}} ; u\left(C_{\text {ref }}\right)_{\text {rel }}=\frac{u\left(C_{\text {ref }}\right) \times 100}{\text { valoratribuido }} \\
\bar{u}\left(C_{r e f}\right)=\sqrt{\frac{\sum u\left(C_{r e f}\right)_{r e l}^{2}}{10}}=0,14 \%\end{array}$ \\
\hline & & $u_{\text {tend }}=\sqrt{\left(R M S_{\text {tend }}\right)^{2}+\left[\bar{u}\left(C_{\text {ref }}\right)\right]^{2}}=\sqrt{(0,51)^{2}+(0,14)^{2}}=0,53 \%$ \\
\hline 5 & $\begin{array}{l}\text { Calcular a } \\
\text { incerteza-padrão } \\
\text { combinada }\end{array}$ & $\begin{array}{c}u_{c}=\sqrt{u(x)^{2}+u_{\text {tend }}^{2}} \\
u_{c}=\sqrt{(0,56)^{2}+(0,53)^{2}}=0,77 \%\end{array}$ \\
\hline 6 & $\begin{array}{l}\text { Calcular a incerte- } \\
\text { za expandida }\end{array}$ & $U_{T P}=2 \cdot u_{c}=2 \times 0,77=1,5 \%$ \\
\hline
\end{tabular}

FRX: fluorescência de raios $X$
Tabela 3

Etapas do cálculo da incerteza de medição na determinação de $\mathrm{SiO}_{2}$ por fluorescência de raios $X$ com resultados obtidos no teste de proficiência GeoPT para obter a incerteza de tendência.

\section{RESULTADOS E DISCUSSÃO}

A Tabela 4 apresenta os resultados dos cálculos de incerteza obtidos para todos os óxidos de elementos maiores e menores. A segunda até a quarta linhas contêm, respectivamente, os valores médios de 30 resultados analíticos do MRC BRP-1, desvios-padrão e coeficientes de variação. Estes expressam a componente de incerteza de reprodutibilidade do método e do laboratório. Nas linhas a seguir são informados os valores certificados e respectivas incertezas do MRC BRP-1, usadas para calcular a incerteza de tendência conforme as etapas 3 e 4 da Tabela 2. Em seguida, há as incertezas de medição combinadas e expandidas obtidas com os dados do MRC BRP-1. A antepenúltima linha da Tabela 4 contém as incertezas-alvo expandidas ( $\mathrm{k}=2)$, estimadas como $1 / 3$ da incerteza da expressão de Horwitz e calculadas para a fração em massa de cada óxido no MRC. As duas últimas linhas da Tabela 4 contêm, respectivamente, as incertezas de medição calculadas usando dados de dez rodadas do teste de proficiência GeoPT e as incertezas-alvo para o menor valor de fração em massa de cada óxido das dez amostras.

Conforme indicado na Tabela 4, as incertezas de medição $\mathrm{U}_{\mathrm{MRC}}$ calculadas são inferiores ou equivalentes às incertezas-alvo para quase todos os óxidos, exceto para $\mathrm{Fe}_{2} \mathrm{O}_{3}, \mathrm{Na}_{2} \mathrm{O}$ e $\mathrm{K}_{2} \mathrm{O}$. Na Figura 1, as componentes de incerteza de reprodutibilidade $\mathrm{u}(\mathrm{x})$, da tendência $\mathrm{u}_{\text {tend }}$ e a incerteza combinada de cada um dos óxidos são apresentadas graficamente. Para $\mathrm{SiO}_{2}, \mathrm{TiO}_{2}, \mathrm{Al}_{2} \mathrm{O}_{3}, \mathrm{MgO}$ e $\mathrm{K}_{2} \mathrm{O}$, a componente da incerteza da reprodutibilidade de medição contribui predominantemente para o valor da incerteza combinada. Os valores de incerteza que expressam a precisão e a veracidade são semelhantes e contribuem igualmente para o valor da incerteza de medição de $\mathrm{Fe}_{2} \mathrm{O}_{3}$, enquanto para óxidos $\mathrm{MnO}, \mathrm{CaO}, \mathrm{Na}_{2} \mathrm{O}$ e $\mathrm{P}_{2} \mathrm{O}_{5}$ a incerteza da tendência é a prin- 
Tabela 4

Valores médios dos resultados de medição e desvios-padrão, valores certificados e incertezas em valores de fração em massa $(\%, m / m)$ de óxidos de elementos maiores e menores do material de referência certificado BRP-1 e resultados de participação no teste de proficiência GeoPT $T^{\mathrm{m}}$, com incertezas de medição calculadas conforme detalhado nas Tabelas 2 e 3, respectivamente. A incertezas-alvo $U_{\text {alvo }}$ representam $1 / 3$ das incertezas de Horwitz, expandidas para $\mathrm{k}=2$.

\begin{tabular}{|c|c|c|c|c|c|c|c|c|c|c|}
\hline$\%$ & $\mathrm{SiO}_{2}$ & $\mathrm{TiO}_{2}$ & $\mathrm{Al}_{2} \mathrm{O}_{3}$ & $\mathrm{Fe}_{2} \mathrm{O}_{3}$ & $\mathrm{MnO}$ & $\mathrm{MgO}$ & $\mathrm{CaO}$ & $\mathrm{Na}_{2} \mathrm{O}$ & $\mathrm{K}_{2} \mathrm{O}$ & $\mathrm{P}_{2} \mathrm{O}_{5}$ \\
\hline $\bar{x}(\mathrm{n}=30)$ & 50,43 & 3,81 & 12,37 & 15,47 & 0,213 & 3,94 & 7,90 & 2,74 & 1,53 & 0,63 \\
\hline $\mathrm{S}_{\mathrm{R}}=\mathrm{u}(\mathrm{x})$ & 0,28 & 0,027 & 0,11 & 0,14 & 0,002 & 0,04 & 0,04 & 0,04 & 0,03 & 0,004 \\
\hline $\mathrm{u}(\mathrm{x})_{\mathrm{rel}}$ & 0,56 & 0,71 & 0,88 & 0,87 & 0,86 & 1,10 & 0,56 & 1,31 & 1,69 & 0,66 \\
\hline VC & 50,39 & 3,81 & 12,40 & 15,59 & 0,216 & 3,94 & 7,95 & 2,71 & 1,52 & 0,63 \\
\hline U(Cref) & 0,15 & 03 & 0,07 & 0,09 & 3 & 03 & 05 & 3 & 0,01 & 0,01 \\
\hline $\mathrm{u}($ Cref) & 0,08 & 0,02 & 0,04 & 0,05 & 15 & 0,015 & 0,025 & 0,015 & 0,005 & 0,005 \\
\hline $\mathrm{u}(\mathrm{Cref})_{\mathrm{rel}}$ & 0,15 & 0,39 & 0,28 & 0,29 & 0,69 & 0,38 & 0,31 & 0,55 & 0,33 & 0,79 \\
\hline tend $_{\mathrm{rel}}$ & 0,09 & 0,02 & $-0,25$ & $-0,78$ & $-1,36$ & 0,03 & $-0,64$ & 1,25 & 0,57 & 0,10 \\
\hline $\mathrm{s}_{\text {tend }} / \sqrt{n}$ & 0,10 & 0,13 & 0,16 & 0,16 & 0,16 & 0,20 & 0,10 & 0,24 & 0,31 & 0,12 \\
\hline $\mathrm{u}_{\text {tend }}$ & 0,20 & 0,41 & 0,41 & 0,85 & 1,53 & 0,43 & 0,72 & 1,39 & 0,73 & 0,81 \\
\hline$u_{c}$ & 0,60 & 0,82 & 0,97 & 1,2 & 1,8 & 1,2 & 0,91 & 1,9 & 1,8 & 1,1 \\
\hline$U_{M R C}$ & 1,2 & 1,6 & 1,9 & 2,4 & 3,5 & 2,4 & 1,8 & 3,8 & 3,7 & 2,1 \\
\hline$U_{\text {alvo MRC }}$ & 1,5 & 2,2 & 1,8 & 1,8 & 3,4 & 2,2 & 2,0 & 2,3 & 2,5 & 2,9 \\
\hline $\mathrm{U}_{\mathrm{TP}}$ & 1,5 & 4,2 & 2,7 & 3,2 & 7,5 & 3,7 & 3,2 & 4,8 & 5,0 & 7,4 \\
\hline$U_{\text {alvoTP }}$ & 1,5 & 4,1 & 2,0 & 2,8 & 4,1 & 3,1 & 3,2 & 2,8 & 3,4 & 4,5 \\
\hline
\end{tabular}

Valor médio $(\%, \mathrm{~m} / \mathrm{m})$ de $\mathrm{n}=30$ resultados de medição, $\mathrm{s}_{\mathrm{R}}$ : desvio-padrão de reprodutibilidade do laboratório; $\mathrm{u}(\mathrm{x})_{\mathrm{rel}}$ : desvio-padrão relativo; VC: valor certificado; $\mathrm{U}(\mathrm{Cref})$ : incerteza expandida do valor de referência, conforme certificado; $\mathrm{u}(\mathrm{Cref})=$ incerteza do valor de referência; $\mathrm{u}(\mathrm{Cref})_{\text {rel }}$ incerteza relativa do valor de referência; tend rel : tendência relativa; $\mathrm{s}_{\text {tend }} / \sqrt{n}$ : desvio-padrão da média dos resultados do MRC; $u_{\text {tend }}$ : incerteza de tendência; $u_{c}$ : incerteza combinada; $U_{M R C}$ e $U_{T P}$ : incertezas expandidas para o MRC e teste de proficiência, respectivamente ; MRC: material de referência certificado

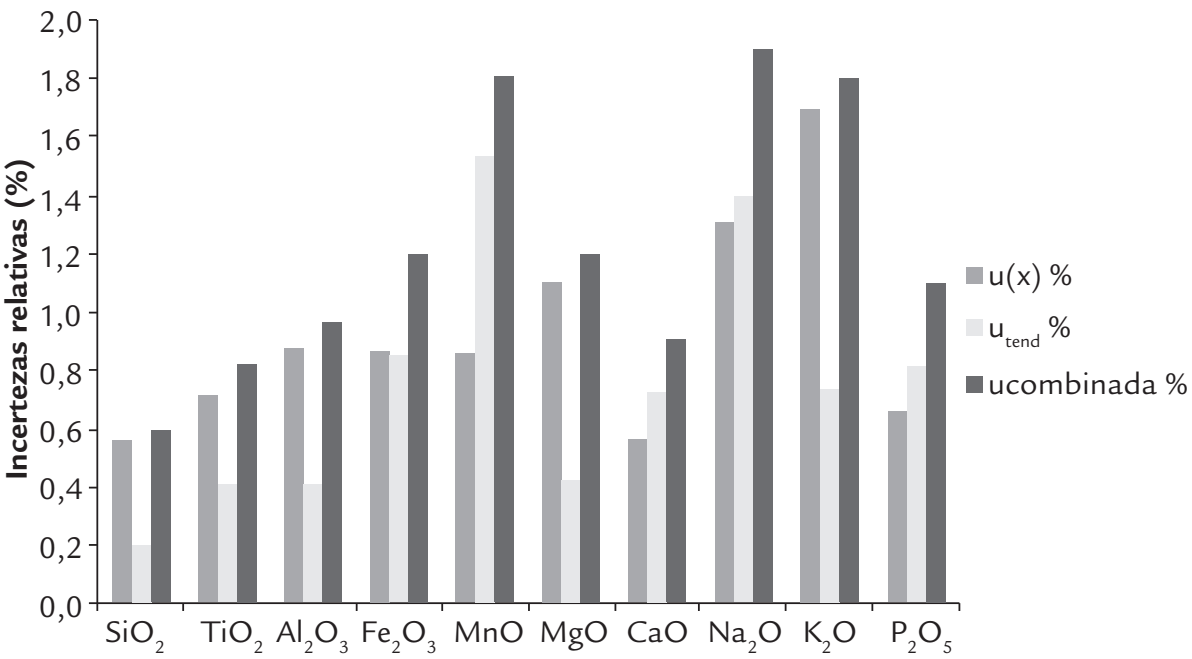

cipal contribuinte para a incerteza de medição. Os valores da Tabela 4 indicam que a componente que mais contribui para a incerteza de tendência para os óxidos $\mathrm{MnO}, \mathrm{CaO}$ e $\mathrm{Na}_{2} \mathrm{O}$ é a própria tendência de medição, enquanto no caso de $\mathrm{P}_{2} \mathrm{O}_{5}$ a incerteza do valor certificado mais influencia no valor da incerteza de tendência.

Os dados de incerteza de medição obtidos para o MRC BRP-1 podem ser extrapolados a amostras com composição semelhante. A avaliação da incerteza de medição com os resultados do teste de proficiência, apesar de ter sido quantificada com dados de somente dez amostras, resulta em valores de incerteza que, em princípio, se aplicam a um grupo mais amplo de composição. As incertezas expandidas resultantes são expressivamente maiores que as incertezas obtidas com o MRC, conforme mostra a comparação dos valores de $\mathrm{U}_{\mathrm{MRC}}$ e $\mathrm{U}_{\mathrm{TP}}$ (Tabela 4 e Figura 2). Estas diferenças provêm essencialmente da incerteza de tendência, uma vez que os mesmos valores de incerteza de reprodutibilidade $\mathrm{u}(\mathrm{x})$ foram usados nos dois cálculos. Apesar da incerteza de tendência ter sido elevada, os escores obtidos para os resultados do laboratório nos respectivos GeoPT estiveram dentro dos limites \pm 2 . As incertezas de medição estimadas $U_{\text {TP }}$ são relativamente elevadas se comparadas com as incertezas-alvo calculadas como um terço da incerteza de Horwitz para o menor valor de fração em massa das amostras dos testes GeoPT utilizados e apresentados na última linha da Tabela. Esta comparação é evidente na Figura 2.

A incerteza associada com a reprodutibilidade dos resultados inclui a precisão do método a partir da confecção do disco de vidro junto com a medição instrumental. Ela foi obtida pela análise de 30 replicatas de um único material já moído. Para avaliar a influência da variabilidade introduzida durante a preparação física das amostras a partir da homogeneização, quarteamento e moagem de amostras britadas, bem como da própria variabilidade composicional, utilizou-se dados de duplicatas de amostras reais. Com os 
resultados de 22 amostras duplicadas foram calculados os desvio-padrão de duplicatas e os respectivos coeficientes de variação (Mullins 2003). Os resultados encontram-se na Tabela 5, onde os dados obtidos para o MRC BRP-1 foram novamente incluídos para facilitar a comparação, que é visualizada na Figura 3. Na parte inferior da Tabela 5, sequencialmente há os intervalos de fração em massa das amostras duplicadas, o valor médio de cada óxido nas amostras, o coeficiente de variação dos resultados, os coeficientes de variação calculados pela expressão Horwitz (novamente 1/3) para os valores médios e para os extremos de composição das duplicatas. Os coeficientes de variação $\mathrm{CV}_{\mathrm{MRC}}$ de $\mathrm{SiO}_{2} \mathrm{e}$ $\mathrm{Al}_{2} \mathrm{O}_{3}$, e $\mathrm{K}_{2} \mathrm{O}$ obtidos para o CRM BRP-1 são maiores que os obtidos para as duplicatas $\left(\mathrm{CV}_{\text {duplicatas }}\right)$ (Figura 3). Para os demais óxidos, a precisão dos resultados das duplicatas foi menor que a obtida para as replicatas do MRC, exceto para os resultados de $\mathrm{MgO}$, em que os coeficientes de variação foram equivalentes para os dois conjuntos de dados. As diferenças de precisão foram atribuídas à diferença de composição do grupo de amostras duplicadas em relação ao

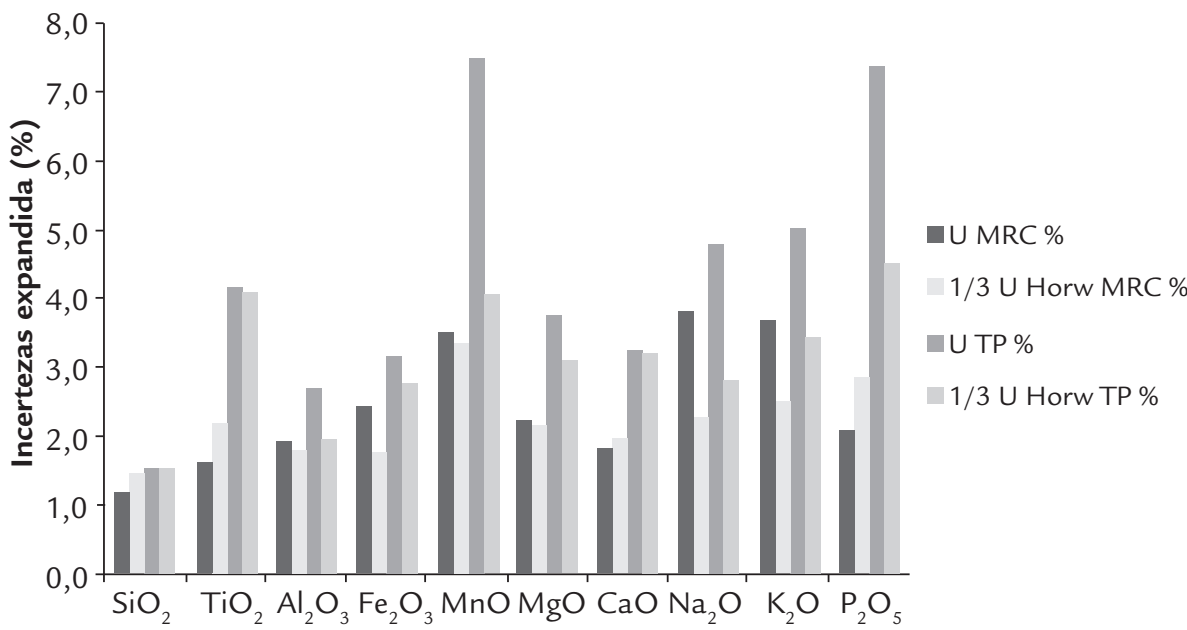

Figura 2

Comparação das incertezas de medição calculadas para os resultados do material de referência certificado BRP-1, com $1 / 3$ da incerteza de Horwitz para os valores de fração em massa do material de referência certificado BRP-1, incertezas de medição calculadas com resultados de dez rodadas do teste de proficiência $\operatorname{GeoPT}^{\mathrm{TM}}\left(\mathrm{U}_{\mathrm{TP}}\right)$ e, novamente, $1 / 3 \mathrm{da}$ incerteza de Horwitz para os menores valores de fração em massa presentes nas amostras GeoPT ${ }^{\mathrm{m}}$ consideradas

MRC: material de referência certificado

\begin{tabular}{ccccccccccc}
\hline$\%$ & $\mathrm{SiO}_{2}$ & $\mathrm{TiO}_{2}$ & $\mathrm{Al}_{2} \mathbf{O}_{3}$ & $\mathrm{Fe}_{2} \mathbf{O}_{3}$ & $\mathbf{M n O}$ & $\mathbf{M g O}$ & $\mathbf{C a O}$ & $\mathbf{N a}_{2} \mathbf{O}$ & $\mathbf{K}_{2} \mathbf{O}_{c}$ & $\mathbf{P}_{2} \mathbf{O}_{5}$ \\
\hline $\bar{x}(\mathrm{n}=30)$ & 50,43 & 3,81 & 12,37 & 15,47 & 0,213 & 3,94 & 7,90 & 2,74 & 1,53 & 0,63 \\
\hline $\mathrm{CV}_{\mathrm{MRC}}$ & 0,56 & 0,71 & 0,88 & 0,87 & 0,86 & 1,10 & 0,56 & 1,31 & 1,69 & 0,66 \\
\hline \multicolumn{8}{c}{ Duplicatas } \\
\hline[]$_{\text {Duplicatas }}$ & $40-97$ & $0,07-3,6$ & $0,9-22$ & $0,6-43$ & $0,01-0,25$ & $0,02-8,7$ & $0,01-23$ & $0,01-4,3$ & $0,01-11$ & $0,02-1,4$ \\
\hline $\bar{x}$ Duplicatas $=\bar{X} \alpha$ & 62,47 & 0,98 & 12,12 & 9,05 & 0,10 & 2,50 & 4,45 & 1,82 & 2,43 & 0,21 \\
\hline $\mathrm{CV}_{\text {duplicatas }}$ & 0,39 & 1,0 & 0,67 & 1,8 & 4,7 & 1,1 & 0,80 & 2,2 & 1,0 & 2,0 \\
\hline $\mathrm{CV}_{\text {Halvo }}$ & 0,7 & 1,3 & 0,9 & 1,0 & 1,9 & 1,2 & 1,1 & 1,2 & 1,2 & 1,7 \\
\hline $\mathrm{CV}_{\text {Halvo[j }}$ & $0,8-0,7$ & $2,0-1,1$ & $1,3-0,8$ & $1,5-0,9$ & $2,7-1,7$ & $2,4-0,8$ & $2,7-0,8$ & $2,7-1,1$ & $2,7-0,9$ & $2,3-1,3$ \\
\hline
\end{tabular}

MRC: material de referência certificado; CV: coeficiente de variação

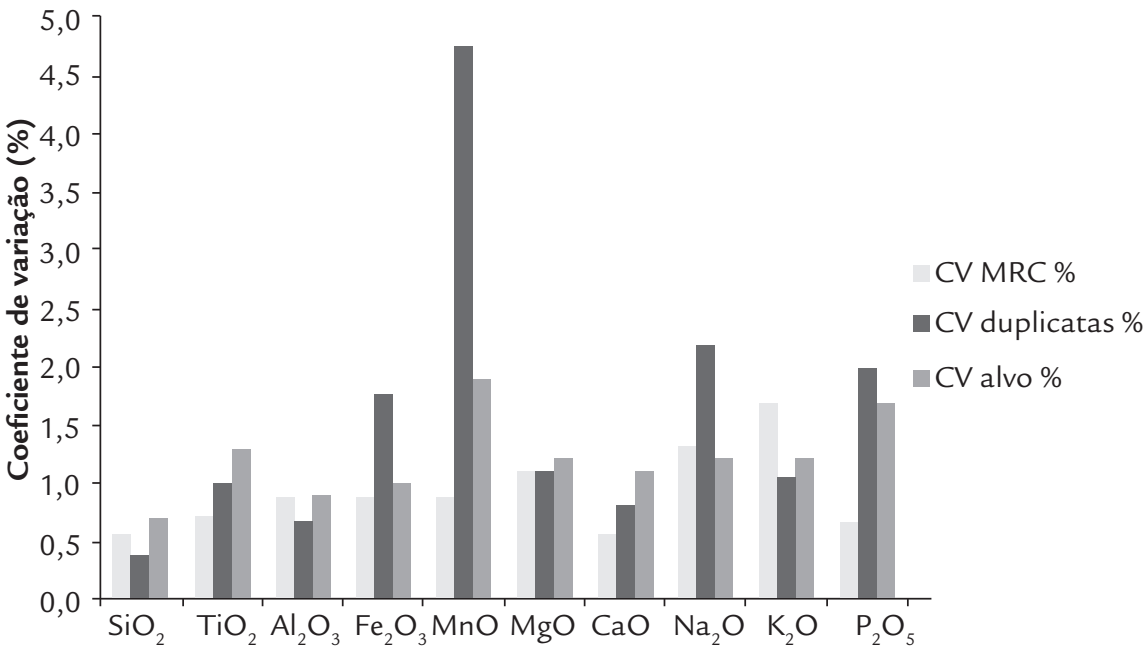

MRC: material de referência certificado; $\mathrm{CV}$ : coeficiente de variação
Tabela 5

Precisão intermediária dos resultados dados pelo coeficiente de variação de replicatas do material de referência certificado e de duplicatas de amostras a partir da etapa de quarteamento. Abaixo da linha duplicatas estão indicados os extremos de composição das amostras $(\%, m / m)$, os respectivos valores médios, os coeficientes de variação calculados e alvo dos valores médio e extremos de composição.

Figura 3

Comparação dos coeficientes de variação obtidos na análise de 30 porções-teste do material de referência certificado BRP-1 e de 22 amostras duplicadas a partir da etapa de quarteamento e coeficientes de variação alvo calculados como $1 / 3 \mathrm{da}$ expressão de Horwitz para os valores de fração em massa equivalente à composição média das amostras duplicadas. 
MRC, conforme indicam os valores extremos e médios da Tabela 5 , comparados à composição do MRC. Para avaliar se os valores elevados de $\mathrm{CV}_{\text {duplicatas }}$ poderiam ser justificados pela variabilidade composicional das 22 amostras duplicadas, a expressão de Horwitz foi usada para calcular o coeficiente de variação como $1 / 3 \sigma_{\text {Hrel }}$ para a fração em massa média dos óxidos no conjunto de duplicatas. Os resultados da Tabela 5 indicam que os $\mathrm{CV}_{\mathrm{H} \text { alvo }}$ são menores que os $\mathrm{CV}_{\text {duplicatas }}$ para $\mathrm{Fe}_{2} \mathrm{O}_{3}, \mathrm{MnO}, \mathrm{Na}_{2} \mathrm{O}$ e $\mathrm{P}_{2} \mathrm{O}_{5}$. A duplicação foi realizada na etapa do quarteamento e, portanto, inclui uma variável importante ausente nos resultados de replicatas do MRC (Vendemiatto \& Enzweiler 2003) e não prevista pela expressão de Horwitz.

Os cálculos de incerteza com conjuntos de dados de controle de qualidade servem também para reavaliar métodos analíticos de forma mais robusta do que a avaliação diária ou de curto prazo, normalmente realizada durante a validação. Os resultados utilizados são todos válidos segundo os critérios de aceitação aplicados, mas os cálculos de incerteza

\section{CONCLUSÕES}

As incertezas de medição obtidas com os resultados das replicatas são menores do que as obtidas com dados dos testes de proficiência. As incertezas de medição obtidas com os resultados das replicatas foram predominantemente menores que as incertezas-alvo propostas e o contrário foi observado para as incertezas obtidas com os dados GeoPT. Em parte, essas diferenças devem-se ao número significativamente menor de dados usados dos testes de proficiência (MRC, $\mathrm{n}=30, \mathrm{x}, \mathrm{Geo} P T, \mathrm{n}=10$ ), mas a variação composicional das amostras utilizadas é um aspecto que não deve ser menosprezado.

A quantificação das incertezas de medição de elementos maiores e menores de silicatos determinados por FRX a partir de replicatas de resultados de análises de um MRC foi útil para conhecer as magnitudes relativas das incertezas mostram que, para alguns óxidos, pequenas modificações no método de medição instrumental podem resultar em incertezas de medição menores. As duas ações mais imediatas que podem ser introduzidas são aumentar um pouco os tempos de contagem (os tempos usados são fixos) de $\mathrm{Fe}, \mathrm{Mn}, \mathrm{K}$ e rever as curvas de calibração desses elementos. Esta última ação, foi aplicada aos dados de MnO no MRC BRP-1 após testar existência de tendência nos resultados com a expressão IMédia das medições - Valor de referêncial $\leq 2 *\left(\left(s_{R}{ }^{2} / n\right)+u(C r e f)^{2}\right)^{1 / 2}$. Pequenos ajustes na curva de calibração de Mn produziram resultados para o MRC com valor médio mais próximo do valor certificado e diminuição das incertezas de tendência, combinada e expandida. Esta correção foi aplicada aos dados do MCR para $\mathrm{MnO}$, mas não aos resultados do GeoPT, e por isso a incerteza $U_{T P}$ é elevada e supera a respectiva incerteza-alvo. A reavaliação completa das condições de medição do $\mathrm{Mn}$, assim como de outros elementos, antes de uma nova calibração do método no espectrômetro, permitiria avaliar as consequências das mudanças propostas.

de reprodutibilidade e tendência e propor possíveis ações para minimizá-las. Mas quais são os valores de incertezas de medição requeridas pelos usuários de dados analíticos? Elas dependem do objetivo do estudo e tipos de amostras analisadas, e se conhecidas poderiam ensejar a revisão dos métodos com o objetivo de torná-los mais robustos. Os resultados obtidos indicam que a quantificação consistente da incerteza de medição pelo método top down idealmente deveria ser realizada com resultados analíticos de diferentes MRC com composições típicas da matriz em questão. No entanto, apesar dos muitos MR de rochas disponíveis, poucos são certificados, e raramente um laboratório pode dispor da quantidade requerida dos MRC para avaliar a veracidade dos métodos empregados, conforme preconizado nos requisitos metrológicos.

\section{REFERÊNCIAS}

Adams C., Cammann K., Deckers H.A., Dobkowski Z., Holcombe D., LaFleur P.D., Radvila P., Rohrer C., Steck W., Vermaercke P. 1998. Quality Assurance for Research and Development and Non-routine Analysis.CITAC/ EURACHEM ISBN: 094892611 2. Disponível em: http://www.citac.cc/ Acessado em 19 nov. 2012.

Cotta A.J.B, Enzweiler J., Nardy A.J.R. 2008. Certificado de análise do material de referência BRP-1 (Basalto Ribeirão Preto). Geochimica Brasiliensis, 22:113-8.

EURACHEM/CITAC Guide CG4 2012. Quantifying Uncertainty in Analytical Measurement. Ellison S.L.R. \& Williams A. (editores) 3. ed. Disponível em: http://www.eurachem.org. Acessado em 19 nov. 2012.

Hibbert D.B. 2007. Quality Assurance for the Analytical Chemistry Laboratory. Oxford University Press, Nova York, 306 pp.

Horwitz W., Kamps L.R., Boyer K.W. 1980. Quality assurance in the analysis of foods for trace constituents. Journal of AOAC International, 63:1344-1354. 
Kane J.S., Potts P.J., Wiedenbeck M., Carignan J. e Wilson S. 2003. International Association of Geoanlysists' Protocol for the certification of geological and environmental reference materials. Geostandards Newsletter, 27:227-244.

Kirkup L. \&Frenkel B. 2006. An Introduction to Uncertainty in Measurement. Cambridge University Press, Cambridge, 233 pp.

Menditto A., Patriarca M. e Magnusson B. 2007. Understanding the meaning of accuracy, trueness and precision. Accreditation and Quality Assurance, $12: 45-47$.

Magnusson B., Näykki T., Hovind H., Krysell M. 2008. Nordtest TR 537, Handbook for calculation of measurement uncertainty in environmental laboratories, $3^{a}$.Ed., Disponível em: http://www.nordtest.info. Acessado em 17 nov. 2012.

Mullins E. 2003. Statistics for the Quality Control Chemistry Laboratory. Royal Society of Chemistry, Cambridge, $455 \mathrm{p}$.

ISO Guide 98-3 (2008) Uncertainty of Measurement - Part 3: Guide to the Expression of Uncertainty in Measurement, 120 p. Disponível em: http://www. iso.org/sites/JCGM/JCGM-introduction.htm. Acessado em 28 set. 2010.

Thompson M., Potts P.J., Kane J.S., Webb P.W. 1996. GeoPT1. International proficiency test for analytical geochemistry laboratories-Report on round 1 . Geostandards Newsletter, 20:295-325.

Vendemiatto M.A. \& Enzweiler J. 2001. Routine control of accuracy in silicate rock analysis by X-ray fluorescence spectrometry. Geostandards Newsletter, 25:283-291.

Vendemiatto M.A. \& Enzweiler J. 2003. Robustez e versatilidade na análise de amostras geológicas por fluorescência de raios X. Geochimica Brasiliensis, 17:75-80.

VIM3 2012.Vocabulário Internacional de Metrologia: conceitos fundamentais e gerais de termos associados (VIM3 2012). Duque de Caxias, RJ : INMETRO, 2012. 94 p. Traduzido de: International Vocabulary of Metrology: basic and general conceptsand associated terms - JCGM 200:2012. 3a. Ed. 2012. Traduzido por: grupo de trabalho luso-brasileiro. ISBN: 978-85-86920-09-7. Acessado em 19 nov. 2012. 